\title{
Evaluation of machine learning algorithms for classification of primary biological aerosol using a new UV-LIF spectrometer
}

\author{
Simon Ruske ${ }^{1}$, David O. Topping ${ }^{1,2}$, Virginia E. Foot ${ }^{4}$, Paul H. Kaye ${ }^{5}$, Warren R. Stanley ${ }^{5}$, Ian Crawford ${ }^{1}$, \\ Andrew P. Morse $^{3}$, and Martin W. Gallagher ${ }^{1}$ \\ ${ }^{1}$ Centre for Atmospheric Science, SEAES, University of Manchester, Manchester, UK \\ ${ }^{2}$ NCAS, National Centre for Atmospheric Science, University of Manchester, Manchester, UK \\ ${ }^{3}$ Department of Geography and Planning, University of Liverpool, Liverpool, UK \\ ${ }^{4}$ Defence, Science and Technology Lab., Porton Down, Salisbury, Wiltshire, SP4 0JQ, UK \\ ${ }^{5}$ Particle Instruments Research Group, University of Hertfordshire, Hatfield, AL 10 9AB, UK \\ Correspondence to: Simon Ruske (simon.ruske@ postgrad.manchester.ac.uk)
}

Received: 22 June 2016 - Discussion started: 13 July 2016

Revised: 22 November 2016 - Accepted: 4 December 2016 - Published: 3 March 2017

\begin{abstract}
Characterisation of bioaerosols has important implications within environment and public health sectors. Recent developments in ultraviolet light-induced fluorescence (UV-LIF) detectors such as the Wideband Integrated Bioaerosol Spectrometer (WIBS) and the newly introduced Multiparameter Bioaerosol Spectrometer (MBS) have allowed for the real-time collection of fluorescence, size and morphology measurements for the purpose of discriminating between bacteria, fungal spores and pollen.

This new generation of instruments has enabled ever larger data sets to be compiled with the aim of studying more complex environments. In real world data sets, particularly those from an urban environment, the population may be dominated by non-biological fluorescent interferents, bringing into question the accuracy of measurements of quantities such as concentrations. It is therefore imperative that we validate the performance of different algorithms which can be used for the task of classification.

For unsupervised learning we tested hierarchical agglomerative clustering with various different linkages. For supervised learning, 11 methods were tested, including decision trees, ensemble methods (random forests, gradient boosting and AdaBoost), two implementations for support vector machines (libsvm and liblinear) and Gaussian methods (Gaussian naïve Bayesian, quadratic and linear discriminant analysis, the $k$-nearest neighbours algorithm and artificial neural networks).
\end{abstract}

The methods were applied to two different data sets produced using the new MBS, which provides multichannel UVLIF fluorescence signatures for single airborne biological particles. The first data set contained mixed PSLs and the second contained a variety of laboratory-generated aerosol.

Clustering in general performs slightly worse than the supervised learning methods, correctly classifying, at best, only 67.6 and $91.1 \%$ for the two data sets respectively. For supervised learning the gradient boosting algorithm was found to be the most effective, on average correctly classifying 82.8 and $98.27 \%$ of the testing data, respectively, across the two data sets.

A possible alternative to gradient boosting is neural networks. We do however note that this method requires much more user input than the other methods, and we suggest that further research should be conducted using this method, especially using parallelised hardware such as the GPU, which would allow for larger networks to be trained, which could possibly yield better results.

We also saw that some methods, such as clustering, failed to utilise the additional shape information provided by the instrument, whilst for others, such as the decision trees, ensemble methods and neural networks, improved performance could be attained with the inclusion of such information. 


\section{Introduction}

Primary biological aerosol particles (PBAP) such as fungal spores, bacteria and pollen have been linked to global atmospheric processes but their impact remains uncertain. In particular, cloud and precipitation feedback mechanisms are dependent on airborne concentrations and surface properties of the particles. Quantification of the biogeography and seasonal variability of such quantities is vital for better understanding the impacts of atmospheric aerosol on the environment.

It is thought that bacteria, pollen and fungal spores can act as cloud condensation nuclei (CCN) and heterogeneous ice nuclei (IN) (Möhler et al., 2007; Hoose and Möhler, 2012). For example, bacterial species such as Pseudomonas syringae and Erwinia herbicola have been shown to be catalysts for the formation of ice at temperatures as warm as $-2{ }^{\circ} \mathrm{C}$ (Gurian-Sherman and Lindow, 1993). Furthermore, ice nucleation active (INA) bacteria have been recovered from cloud water (Joly et al., 2013), demonstrating that bioaerosols, acting as IN, can be found in the atmosphere, at least where these clouds are present, and therefore may be influencing various atmospheric processes.

Only a few bacterial and fungal species have been shown to be INA at the higher range of sub-zero temperatures and even in these cases only a small amount of cells nucleate at these temperatures, leading some to question the significance of bioaerosols as ice nucleators (Cziczo et al., 2013). However, since ongoing research has led to the discovery of new biological ice nucleators (Huffman et al., 2013), there are likely more INA species to be found and under certain conditions, such as during rainfall especially at warmer temperatures, these particles may be having a much more profound impact than previously thought (Huffman et al., 2013; Hader et al., 2014; Prenni et al., 2013; Tobo et al., 2013).

The above recent research has led to the development of the hypothesis of a bioprecipitation feedback cycle, whereby plants release aerosol containing microorganisms and spores that then act as ice catalysts at warmer temperatures than other more common ice nucleators, such as mineral dusts. This in turn facilitates precipitation, which is beneficial for the growth of plants and microorganisms (Morris et al., 2014). Within such a cycle it may be the case that biological particles initiate secondary ice nucleation processes, also at warmer temperatures (Crawford et al., 2012), leading to more rapid cloud glaciation which may also impact the development of precipitation. Emissions of certain bioaerosols are also predicted to increase in a warming climate (Jacobson and Streets, 2009), resulting in changing patterns of plant and animal disease spread (Kennedy and Smith, 2012).

Whilst the technology for identification and quantification of specific airborne bioaerosols exists, measurements of their concentrations and surface properties remain some way off. Nonetheless, the practicality of long-term, continuous, real-time monitoring and discrimination of at least some of these properties for the more common types has already been demonstrated, e.g. at rural and semi-rural background sites in Germany, Ireland and Finland (Healy et al., 2014; Toprak and Schnaiter, 2013; Schumacher et al., 2013).

Despite the limited observations of the concentrations of bioaerosols, their effects on the outcomes of global and regional aerosol models have been investigated (Spracklen and Heald, 2014; Hummel et al., 2015). In Spracklen and Heald (2014), simulated concentrations of fungal spores and bacteria are used in a global aerosol model from which they conclude that, whilst PBAP contribute very little to average global immersion freezing ice nucleating rates, PBAB dominates ice nucleation at warmer temperatures at certain altitudes. In Hummel et al. (2015), measurements from a number of field sites have also been used to test high-resolution bioaerosol emission models on European regional scales, from which it is suggested that simulated fluorescent biological aerosol particle concentrations based on literature emission parametrisation are lower than the corresponding measured concentrations in key emission regions. As well as further field research, evaluation of the algorithms discussed in this paper could allow for more certainty in the measurements of the concentrations, which would allow for better validation of the above models.

Furthermore, there are other uncertainties which arise from the potential misclassification from interferents, particularly in complex urban environments. Potential nonbiological fluorescent aerosol interferents may include black carbon aerosols from seasonally varying solid fuel sources (Herich et al., 2014). Addition of organic films via deposition of polycyclic aromatic hydrocarbons (PAHs) emitted by vehicle exhausts is another potential interferent, as are common mineral dusts containing fluorescent rare-earth metals. In addition to the compilation of larger data catalogues to help address the issue of interferents (e.g. Hernandez et al., 2016), there also needs to be a focus on testing the effectiveness of approaches to distinguish between particles reliably in real time.

Hierarchical cluster analysis (HCA), an unsupervised learning technique, has been used previously to discriminate between bioaerosol (Robinson et al., 2013; Crawford et al., 2014, 2015). This technique has been shown to be successful in discriminating between various polystyrene latex spheres (PSLs) and has been applied to ambient data where correct classification is unknown. In this paper we extend this research to encompass laboratory samples where correct classification is known in an attempt to evaluate the performance of such algorithms with data that are more similar to that which could be produced during an ambient campaign.

To enhance our study, we also conduct analysis using a range of supervised methods. There are many advantages and disadvantages of supervised methods versus unsupervised methods. Firstly, supervised techniques allow one to choose training data and groupings that better reflect the research problem at hand. For example, for discriminating between 
bacteria, fungal spores and pollen with the aim of studying how they interact with the atmosphere, one could collect various different samples of the different groups and use this to train supervised methods to identify the particles in ambient data. Conversely, the results from the unsupervised methods are dependent on natural differences in the data and cannot be tailored towards a particular application.

Secondly, when faced with a previously unseen particle, the supervised methods may be dependent on the data with which they were trained. Unsupervised methods may offer an advantage in these cases since they are not reliant on training data. Another factor that needs to be considered is the time cost of the different methods. Supervised methods such as decision trees and linear discriminant analysis (LDA) offer much faster alternatives to hierarchical cluster analysis, which would be important when considering real-time applications in the future.

Clearly, supervised methods may offer additional benefits making their study worthwhile, but the laboratory data collected prior to ambient studies will be of paramount importance. Specifically we test 11 methods available in the scikitlearn package (Pedregosa et al., 2011) including decision trees, ensemble methods (random forests, gradient boosting and AdaBoost), two implementations for support vector machines (libsvm and liblinear), Gaussian methods (Gaussian naïve Bayesian, quadratic discriminant analysis (QDA) and LDA) and finally the $k$-nearest neighbours algorithm. In addition we test neural networks provided in the pycaffe package (Jia et al., 2014).

\section{Methods}

In the classification of biological aerosol the primary aim is to attribute a label to each of the particles. Unsupervised learning requires no prior knowledge and splits the particles into different groups using natural differences in the data. Supervised learning takes a subset of the data, which we will call the "training set", and uses this "learn" differences between groups. A testing stage on the remaining data, which we will call the "testing set", is then conducted. The percentage of the testing set correctly classified is then recorded to evaluate how well the method has "learnt" how to distinguish between the groups.

We split the data using five-fold cross validation. Here the data are randomly split into five groups. We then progressively take each group to be the test set and use the remaining four groups to train each of the methods and then record the percentage of the test group that was correctly classified. Finally we average our results over the five tests.

For HCA we varied whether we (a) included both saturated and non-fluorescent data, (b) included saturated data but not non-fluorescent, (c) included only non-fluorescent data but removed saturated data and (d) removed both. We concluded that a particle was non-fluorescent if its eight fluorescence measurements lay within 3 standard deviations of the mean measurements when the instrument was empty. Such filtering is common for previous studies using hierarchical clustering but filtering was not considered for the supervised learning methods since the methods should be able to incorporate some kind of filtering within their own classification schemes. For example when using decision trees, removal of non-fluorescent data would be replicated using branches that split the data based on the fluorescence above and below a certain threshold. We therefore conclude in the case of supervised methods that it is beneficial to allow the method to have full control of how the data are grouped for classification rather than to filter any of the data ourselves.

For some of the methods it is necessary to standardise the data before conducting the analysis. This is the case for cluster analysis (Sect. 2.1), support vector machines (Sect. 2.5) and neural networks (Sect. 2.6). The purpose of standardisation is to consider each of the variables with equal weight. For example, the fluorescent measurements are much larger than the size measurements and for the aforementioned methods this would cause the fluorescent measurements to have more of an influence on the classification than the other variables, which in turn leads to a significant drop in performance. For decision trees and ensemble methods (Sect. 2.2) we do not standardise as each of the variables are considered in isolation so standardisation is not necessary. For the Gaussian methods (Sect. 2.3), standardisation is conducted implicitly when the models are fitted so standardisation again is not necessary. For $K$-nearest neighbours (Sect. 2.4), standardisation is usually recommended but with our initial tests we found that it hindered performance for our data, so for our results this method is produced from unstandardised data. In order to standardise the data we apply the $z$ score to each of the variables since this is the method of standardisation that is most commonly used in the literature (e.g Crawford et al., 2015).

The structure of Sect. 2 is as follows: in Sect. 2.1 we discuss the only unsupervised method we tested - HCA. In Sect. 2.2 we highlight decision trees and ensemble methods encompassing everything from a single decision tree to any method that can be used to combine multiple decision trees in an attempt to create a better classifier (AdaBoost, gradient boosting and random forests). Gaussian methods are introduced in Sect. 2.3; these include any method that fits a Gaussian model to the data for classification, including LDA, QDA and Gaussian naïve Bayesian. In Sect. 2.4 we highlight the $k$-nearest neighbour classifier and in Sect. 2.5 we discuss the main differences between the two implementations of support vector machines. Finally in Sect. 2.6 we discuss neural networks.

\subsection{Hierarchical cluster analysis}

HCA is the only unsupervised method that we tested. Other unsupervised methods such as $k$-means clustering and not 


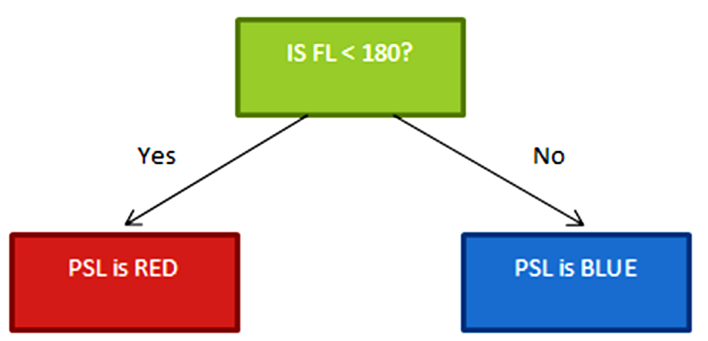

Figure 1. An example of a small decision tree.

considered here since they rely on the user to input the number of clusters, which in an ambient situation is unlikely to be known prior to the analysis. There are seven available linkages in the Fastcluster package (Müllner, 2013, 2011): single (closest point), complete (furthest point), average (average distance), weighted (weighted average distance), ward (minimisation of variance), centroid (difference between means) and median (differences between medians). Prior to this analysis we used the $z$ score to standardise the data.

\subsection{Decision trees and ensemble methods}

When using decision trees, data are split by sorting the data by each variable and using a metric to find the best place to split. An example of a decision tree is given in Fig. 1. In our example there are two groups, blue particles and red particles, and the variable we use to split them is a measurement of fluorescent intensity of the particle. In reality the tree will be much more complicated with many more branches.

To construct the decision tree we consider all possible splits within the data. For example if we had three particles with fluorescent intensity (FL) of 180, 300 and 1400 arbitrary units, we would consider all possible splits to determine the best split for the first branch. For three particles there would be three possible cases for the first branch: FL $>180$, $\mathrm{FL}>300$ and FL $>1400$. Each split then will be evaluated using a criterion to determine how effective the split is to distinguish between the different groups. All of the other variables are then considered in the same fashion and the most effective split for the first branch in the data is selected. The process is then repeated to split the data multiple times, creating a larger tree with many branches. In the case of our example we would have a tree with two splits. When classifying a new particle we simply start at the top of the tree, evaluating the criteria until a conclusion about the particle is made.

Multiple decision trees can be combined to create ensemble classifiers. These classifiers often achieve improvements in one of two ways. Firstly, classifiers such as bagging and random forests take samples of the data and the variables, which are used to produce different decision trees, each capable of classifying a particle. Averaging the classifications made by each tree is then thought to give an overall better re- sult. An alternative approach used by the AdaBoost classifier and the gradient boosting classifier is to begin by weighting all the data equally and over several iterations have decision trees focus on the parts of the data that are being misclassified most often. This can yield an improvement over the single decision tree as the classifier is modified to correct the mistakes that it is making. These ensemble methods could be theoretically used with other classifiers but the simplicity and speed of the decision trees mean that they are most often used. We give further details of the ensemble methods below.

Bagging (Breiman, 1996) is where multiple samples of the data are taken and a different tree is fitted to each of the samples. The samples taken are bootstrap samples, a common statistical technique used to create multiple data sets from one set of data. This can be thought as putting all the samples into a bag, taking out one sample at a time and putting it back into the bag. This is repeated until a new data set which is the same size as the original is obtained. Some samples will have been selected more than once from the bag and others may not get selected at all. This gives a subtly different data set. This can be repeated multiple times in order to create multiple versions of the data set. From each of the samples a decision tree is constructed and the results from the different trees are then averaged to give an overall result. The rationale behind the method is that slight differences in the different versions of the data set will produce different trees and in averaging the results we will get a better estimation of which group the particle belongs to.

Bagging is extended to "random forests" in Breiman (2001). Instead of selecting the best split when constructing any particular tree, a random subset of variables is chosen to build the tree. It is hypothesised that using only a subset of the variables will produce trees that are more independent and thus the improvement from averaging can be larger. Random forests are generally considered to perform better than bagging; hence we do not consider bagging in our analysis.

An alternative method for combining decision trees into an ensemble classifier is AdaBoost (Freund and Schapire, 1995). Here weights are assigned to each of the particles and very small decision trees are fitted to the data. Performance is evaluated using a loss function (exponential loss function) and the data are re-weighted to focus on particles that are being misclassified most often. Gradient boosting is a generalisation of the AdaBoost algorithm to allow for different loss functions.

\subsection{Gaussian-based methods}

An alternative approach to solve the classification problem is to fit multivariate normal distributions to each of the groups within the training data. This distribution is a generalisation of the normal distribution for one variable and depends on the means and covariance of the different variables.

Different assumptions of how the covariance between the variables behaves leads to different classifiers. If one places 


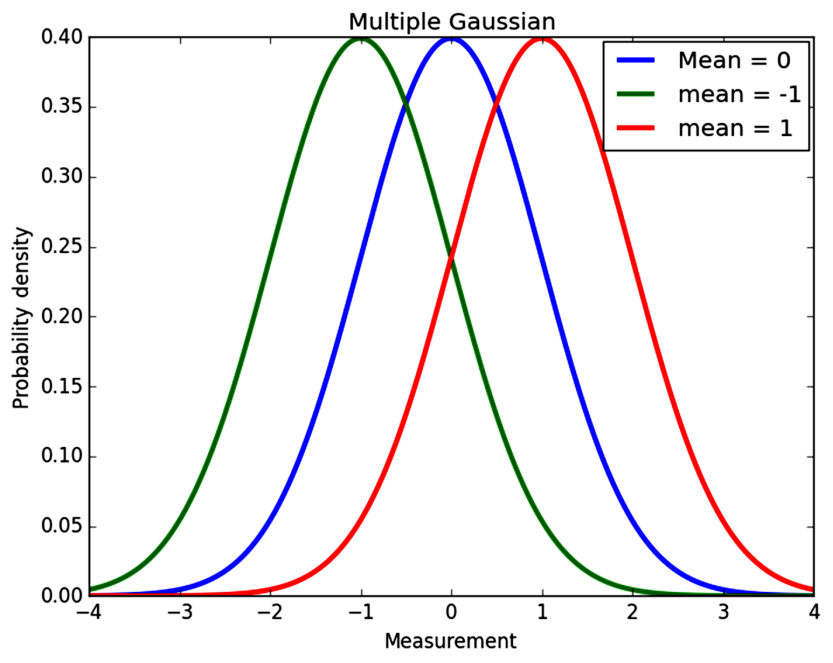

Figure 2. An example of three fitted normal distributions.

no requirements on the covariance this results in QDA. The assumption that each of the groups vary equally results in LDA and finally making the assumption that each of the variables are independent of each other results in Gaussian naïve Bayesian.

Once the normal distributions are fitted we can classify new particles by calculating the probability that the particle comes from each of the groups and assigning it to the group that it is most likely to have come from.

As an example, in Fig. 2 we have plotted normal distributions for three groups. If we were to observe a value of $x=0$ then the particle would be assigned to the blue group as the probability that the particle comes from the blue group is higher than that for the red and for the green.

\section{$2.4 K$-nearest neighbour classification (KNN)}

This method does not require a training step; instead, to classify a previously unseen particle the particle is compared to each of the particles in the training set and the $k$-nearest neighbours in the training set to the previously unseen particle are recorded. The previously unseen particle is then attributed to the same group as the majority of its nearest neighbours. This method can be regarded as recognition rather than learning as it classifies a particle simply on how similar it is to particles that it has seen in the training data.

\subsection{Support vector machines}

A practical guide to support vector classification is given in Hsu et al. (2003). The method transforms the data to a higherdimensional space and then splits the data using a linear decision function (Cortes and Vapnik, 1995). In two dimensions this would be a line, in three a plane, etc. In two dimensions, points to one side of the line are classified as coming from one group; points on the other side of the line are classified as coming from the other group. Points to either side of the line correspond to positive and negative values of the decision function respectively. The line is selected on the basis of how well it splits the data without giving too much precedence to outliers.

In order to generalise this methods to multiple groups there are two methods: one-vs.-rest and one-vs.-one. One-vs.-rest involves fitting a support vector machine for each of the groups against the rest of the groups and then attributing new particles to the group with the highest value of the decision function. One-vs.-one fits a classifier to each pair of groups and then uses a voting scheme to attribute previously unseen particles to a group. LinearSVC (linear support vector classification) uses the one-vs.-rest strategy whereas SVC (support vector classification) uses the one-vs.-one strategy.

How the data are transformed to a higher-dimensional space is dependent on the kernel chosen. There are two implementations within scikit-learn (Pedregosa et al., 2011) that can be used for support vector machines: SVC and LinearSVC. The former allows many different kernels, whereas the latter is a faster version of the first but is limited to the linear kernel only. We test SVC using the RBF (radial basis function) kernel and use linearSVC for the linear kernel.

The SVC implementation has parameters $\gamma$ and $C$. Since $\gamma$ is a specific parameter for the RBF kernel, LinearSVC only requires the input of the value of $C$. Using a sample of $10 \%$ of the data, we test the values of $C$ equal to $1,10,100$ and 1000 and in the case of the SVC function we test all possible combinations of $C$ with $\gamma$ equal to $0,1,10,100$ and 1000 . The values are selected to test a wide range of possible values of each of the parameters to allow for appropriate values to be selected. In future, it might be possible to get better performance by either conducting this initial parameter selection on a larger sample of the data or testing more values, but within the scope of this paper we are intending to select parameters that perform fairly well, which should give us an appropriate estimation on the effectiveness of the method. The values which perform best are used with the five-fold cross validation to form our final result.

\subsection{Artificial neural networks}

Artificial neural networks are statistical models inspired loosely by neurons within the brain. They have been shown to be particularly effective for complex problems such as digit classification (LeCun et al., 1998). As with support vector machines, it is recommended that each of the variables are standardised using the $z$ score to ensure that each of the variables are given equal weighting when training the neural network.

A neural network consists broadly of three components. Firstly, an input layer, which is the fluorescence, size and shape for each of the particles for the data presented. Next we have hidden layers. The network may contain one or many hidden layers and each hidden layer will contain many hid- 
den units, or neurons. Synapses connect the input layer to the hidden layer(s) and from the hidden layer(s) to the output layer. The synapses have a simple job of multiplying the input value by a weight and producing an output value. The neurons will sum the outputs of the synapses and apply an activation function. Finally we have an output layer. For our data this will be the classification of the particle, e.g. bacteria or blue PSL. For this paper we experimented with using one hidden layer with 10 hidden units and two hidden layers with 500 and 10 units respectively.

Initially the weights in the network are set randomly using a normal distribution with mean 0 and standard deviation of 0.01. Since the weights have been initialised randomly at this point the network will perform very poorly. However, as data are passed through the network, the weights are adjusted to minimise a loss function. Once training is completed the weights should better reflect the learning task and then the network is used to classify the testing set.

The scikit-learn package does not currently contain an implementation for neural networks but is undergoing development and will likely do so in the future. For this paper we elect to use pycaffe (Jia et al., 2014), which is a fast package for implementing a variety of different neural networks.

\section{Instrumentation}

The Multiparameter Bioaerosol Spectrometer (MBS) is a development of the Wideband Integrated Bioaerosol Spectrometer (WIBS) technology developed by the University of Hertfordshire (Kaye et al., 2005). Both instruments are designed to acquire data relating to the size, shape and intrinsic fluorescence of individual airborne particles and use these data to detect and potentially classify those particles that are of biological origin. However, whereas the WIBS instrument records particle fluorescence over just two wavebands, approximately $310-400$ and $420-650 \mathrm{~nm}$ (corresponding to the maximum emissions from tryptophan and NADH), the MBS records the fluorescence over eight equal wavelength bands from approximately 310 to $640 \mathrm{~nm}$. This is likely to provide better discrimination between biological particles and "interferent" non-biological particles that may exhibit similar fluorescence properties. Similarly, while WIBS uses a simple 4 pixel detector to assess particle shape from the particle's spatial light scattering pattern (Kaye et al., 1996; Kaye, 1998), the MBS uses an arrangement of two 512 pixel CMOS detector arrays to record high-resolution details of the particle's spatial light scattering pattern, allowing both the macroscopic shape of the particle and potentially particle surface characteristics to be determined. Again, this can enhance the prospects of particle classification and reduces false-positive bio-particle detection. The key elements of the MBS are shown in Fig. 3.

The MBS draws ambient aerosol through an inlet tube at a rate of approximately $1.5 \mathrm{~L} \mathrm{~min}^{-1}$. Part of this flow is fil- tered and used both as a "bleed" flow (to maintain cleanliness of the inner optical chamber) and as a "sheath" flow which surrounds and constrains the remaining "sample" flow. Particles carried in the remaining $300 \mathrm{~mL} \mathrm{~min}^{-1}$ sample flow are forced to pass in single file through the sensing volume, defined by the intersection between the particle detection laser beam (see below) and the sample airflow column.

Each particle carried in the sample airflow is initially detected by a low-power laser beam $(12 \mathrm{~mW}$ at $635 \mathrm{~nm})$. The light scattered from the laser pulse is collected by the lens assembly shown at the upper-right of Fig. 3 and a small proportion of the light is directed by a pellicle beam splitter to the photodiode trigger detector. The voltage output pulse of this detector is proportional to the intensity of light falling on it and is used to size the particle. The trigger signal also initiates the firing of a second, high-power, pulsed laser $(250 \mathrm{~mW}$ at $637 \mathrm{~nm}$ ) that irradiates the particle with sufficient intensity to allow elements of the particle's spatial light scattering pattern, which relates to particle morphology and orientation (Kaye, 1998), to be captured by the arrangement of two CMOS linear detector arrays.

About $10 \mu$ s after particle detection, the UV xenon source illuminates the particle for approximately $1 \mu \mathrm{s}$ with an intense UV pulse at $280 \mathrm{~nm}$ wavelength. The resulting fluorescent light from the particle is collected by two spherical mirrors and directed through to the spectrometer optics. The fluorescence spectrum, covering $310-650 \mathrm{~nm}$, is recorded by the eight-channel photomultiplier tube and the information is digitised and recorded by the electronics control unit. The particle then passes out of the chamber and the system is rearmed. The total measurement process takes $30 \mu \mathrm{s}$. Despite the fact that the system is capable of counting particles at a rate greater than 1000 per second, the limiting factor is the xenon recharge time (approximately $5 \mathrm{~ms}$ ), which reduces the data acquisition rate to approximately 100 particles a second (this corresponds to measuring all particles to a concentration of $2 \times 10^{4}$ particles $\mathrm{L}^{-1}$.

Figure 4 below illustrates the type of data produced by the MBS for an individual airborne particle. The particle fluorescence spectrum, excited by the $280 \mathrm{~nm}$ UV xenon flash, is denoted by the blue bars in the left-hand plot. The red bars represent the average fluorescence values for the previous 25 particles measured. The right-hand plot shows the relative intensity patterns of scattered light from the particle when illuminated by the high-power $637 \mathrm{~nm}$ laser pulse. The red and green plots, extending left and right from the centre, correspond to the relative intensities of light falling onto the two linear CMOS detector arrays shown in Fig. 3. The symmetry (or asymmetry), form and magnitude of these intensity distributions are related to particle shape and surface structure and are therefore characteristic of the morphology of the illuminated particle, thus offering additional parameters by which the particle may be classified. 


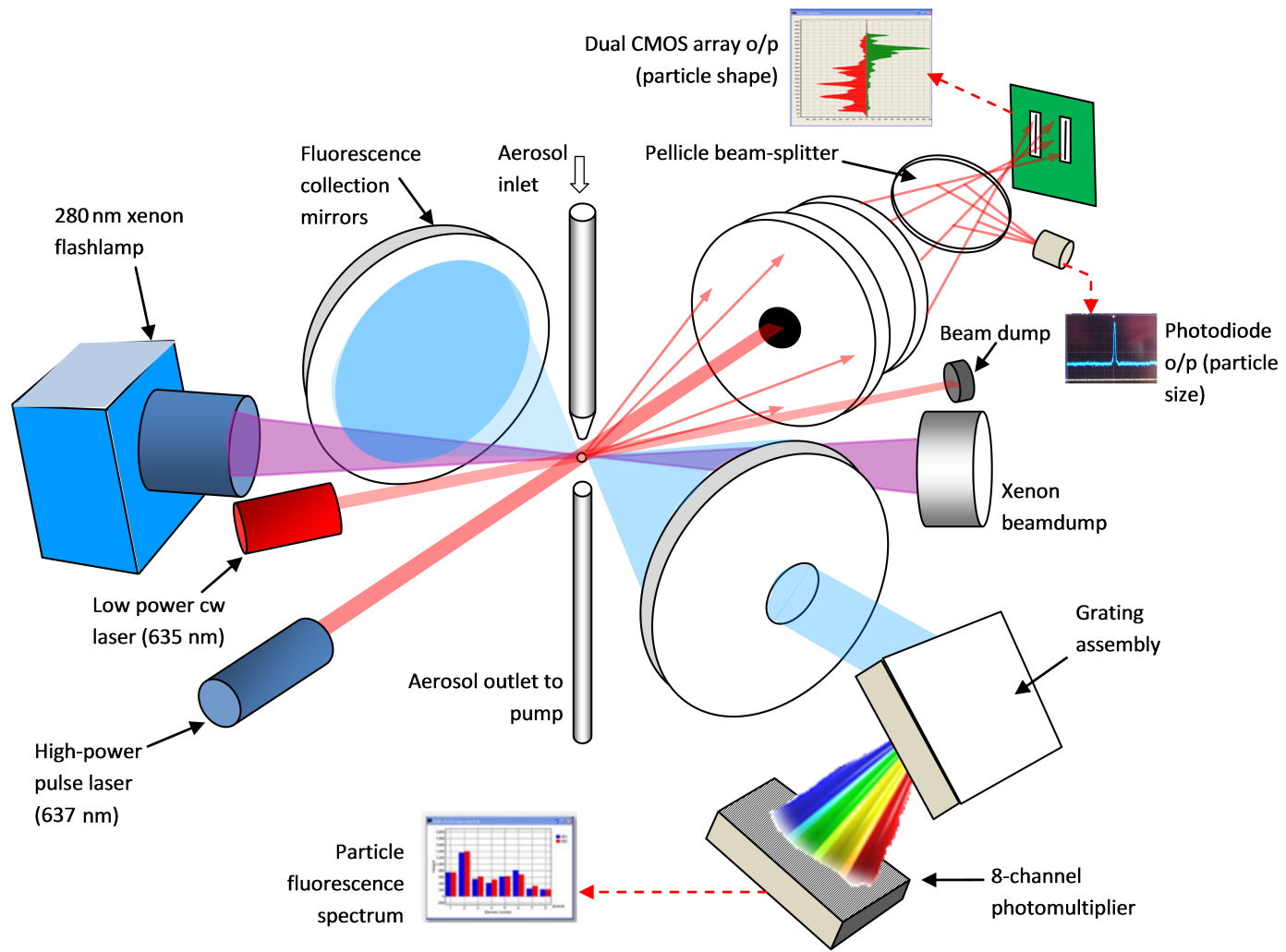

Figure 3. Schematic diagram of the Multiparameter Bioaerosol Spectrometer.
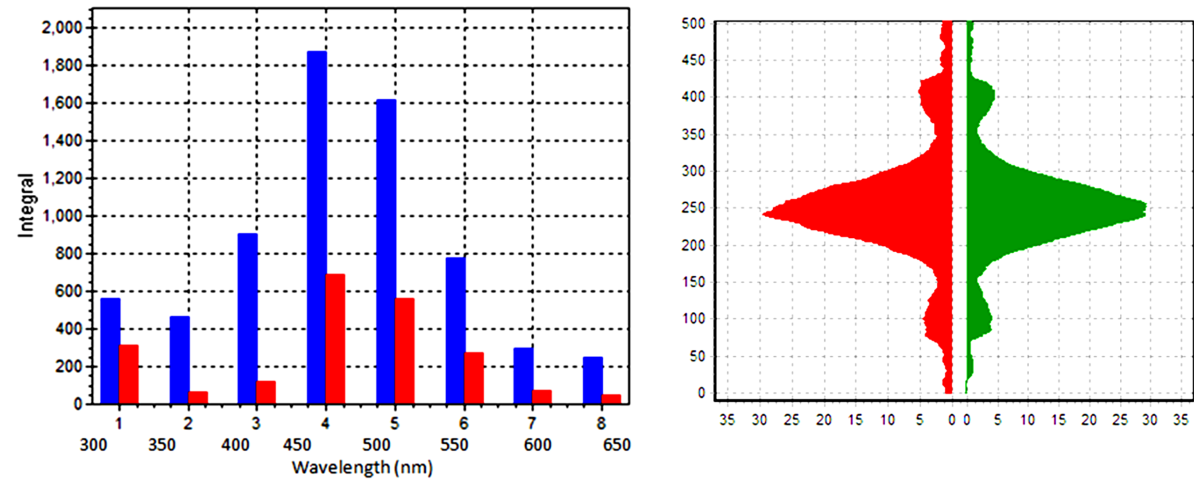

Figure 4. Typical fluorescence spectral data (left) and spatial light scattering data (right) recorded from a single aerosol particle by the MBS instrument.

\section{Data}

In order to evaluate the performance of the various different methods we use two different data sets. For each of the data sets we have included a parallel coordinate plot to allow the reader to see on average how each of the groups differ in their fluorescent intensity and size (see Figs. 5 and 6).

\subsection{Polystyrene latex spheres}

From Fig. 5 it should be clear that the PSLs should be highly separable by eye. This data set provides a benchmark of the simplest separation task. We would expect a good classification technique to perform well with this data set.

Six groups of spheres, five of which have been doped in colouring, of varying sizes are used. Details of the sizes and the doping of the different groups are given in Table 1. These data are very similar to those used in Crawford et al. (2015), 


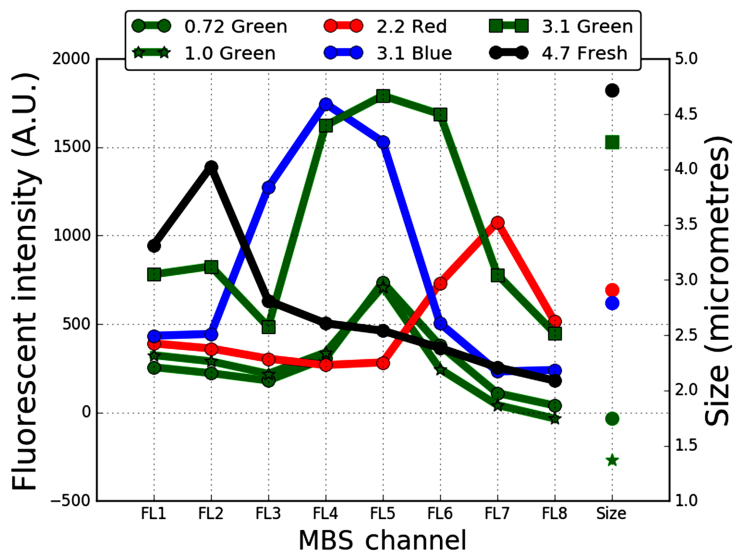

Figure 5. Average fluorescent intensity given in arbitrary units (AU) for the eight fluorescent channels and the size given in micrometres for the PSL data.

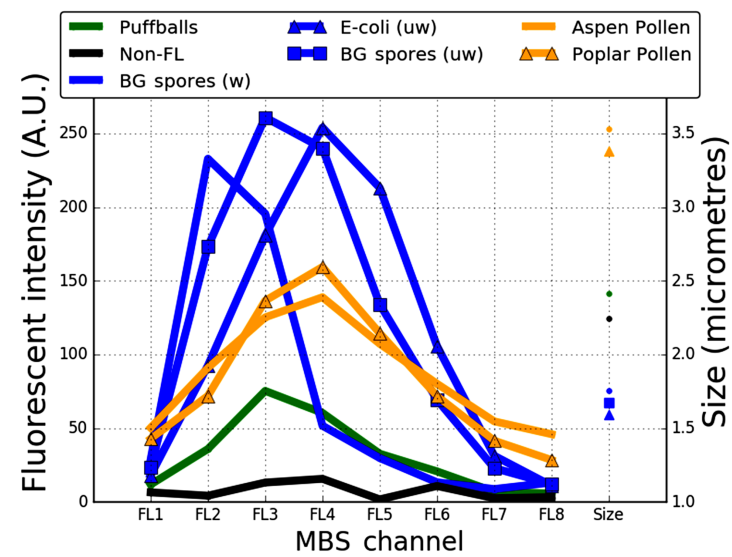

Figure 6. Average fluorescent intensity given in arbitrary units (AU) for the eight fluorescent channels and the size given in micrometres for the laboratory data. The fluorescent signatures for the paper mulberry sample are not included in the figure as the particles are much larger and more fluorescent than the remaining samples and their inclusion would cause the graph to be uninterpretable.

in which hierarchical agglomerative clustering was shown to effectively discriminate particles of this kind.

\subsection{Laboratory data}

The laboratory data used here are an attempt to provide challenging aerosol particles that are more representative of those occurring naturally in the environment and between which a bioaerosol sensor will need to discriminate. These data contain examples of various different fungal spores, pollen, bacteria and non-fluorescent material that might be found within ambient data.

The materials listed in Table 2 were aerosolised into a large, clean HEPA filtered containment chamber (incorporating a recirculation fan), from which the aerosol inlet of
Table 1. Sample sizes for PSLs.

\begin{tabular}{llr}
\hline Size & Doping & $\begin{array}{r}\text { Number of } \\
\text { particles }\end{array}$ \\
\hline $2.2 \mu \mathrm{m}$ & Red & 8704 \\
$3.1 \mu \mathrm{m}$ & Green & 9651 \\
$3.1 \mu \mathrm{m}$ & Blue & 10076 \\
$0.72 \mu \mathrm{m}$ & Green & 2702 \\
$1 \mu \mathrm{m}$ & Green & 5274 \\
$4.17 \mu \mathrm{m}$ & None & 4351 \\
\hline
\end{tabular}

the MBS sensor drew the measurement samples. Liquids and suspensions were nebulised using a medical mini-nebuliser (e.g. Hudson RCI Micro-Mist nebuliser), while the dry materials were aerosolised directly from small quantities of powder using a filtered compressed air jet.

The Bacillus atrophaeus (BG) and E. coli bacteria were generated from suspensions in L-broth growth media, so these aerosols also contain particles of L-broth. Some of the BG spores were also washed before use (by filtering the suspension and re-suspending the spores in distilled water) to obtain relatively clean aerosolised spores.

Measurements of a rye grass pollen sample were taken but only consisted of approximately 50 particles, substantially less than the other samples, and so were removed.

The remaining particles were split into four broad groups: bacteria, fungal spores, pollen and non-fluorescent material. Details of the sample sizes and group classifications are given in Table 2.

\section{Results}

\subsection{General results}

After being split into training and testing data, as outlined in Sect. 2, the proportion of the testing data correctly classified for each of the supervised methods for each of the data sets is given in Figs. 7 and 8. In the case of the unsupervised method (HCA) it was not necessary to split the data into training and testing sets. Instead we applied the algorithm with all seven available linkages to all the particles. The results for which, for ease of comparison, are also given in Figs. 7 and 8.

We have also provided a subset of our time results. In Fig. 9 we have the training and testing times for the supervised methods and the full time taken for the HCA for the full mixed PSL data. The timings for the reduced data set and for the laboratory-generated aerosol are omitted as they show similar patterns. Note in particular that our training set is four times bigger than our testing set since we have used five-fold cross validation (see Sect. 2).

When applying HCA we investigated whether removal of non-fluorescent particles and saturated particles gave better performance. For the PSL data set the best results were 
Table 2. Classification, generator method and sample size of different samples from the laboratory data.

\begin{tabular}{lllr}
\hline Material & Generator method & Classification & No. of Particles \\
\hline Bacillus atrophaeus spores (unwashed, in L-broth) & Mini-nebuliser & Bacteria \\
Phosphate-buffered saline & Nebuliser & Non-fluorescent & 1831 \\
Puff ball spores & Dry & Fungal spores & 1388 \\
Poplar pollen & Dry & Pollen & 2607 \\
Paper mulberry pollen & Dry & Pollen \\
Aspen pollen & Dry & Pollen \\
Bacillus atrophaeus spores (washed, in distilled water) & Mini-nebuliser & Bacteria \\
Escherichia coli MRE 162 (E. coli) cells (unwashed, in L-broth) & Mini-nebuliser & Bacteria \\
Sodium chloride (fluka no. 71 376) 1\% aqueous solution & Mini-nebuliser & Non-fluorescent \\
Fuller's earth dust & Dry from mini-nebuliser & Non-fluorescent \\
\hline
\end{tabular}
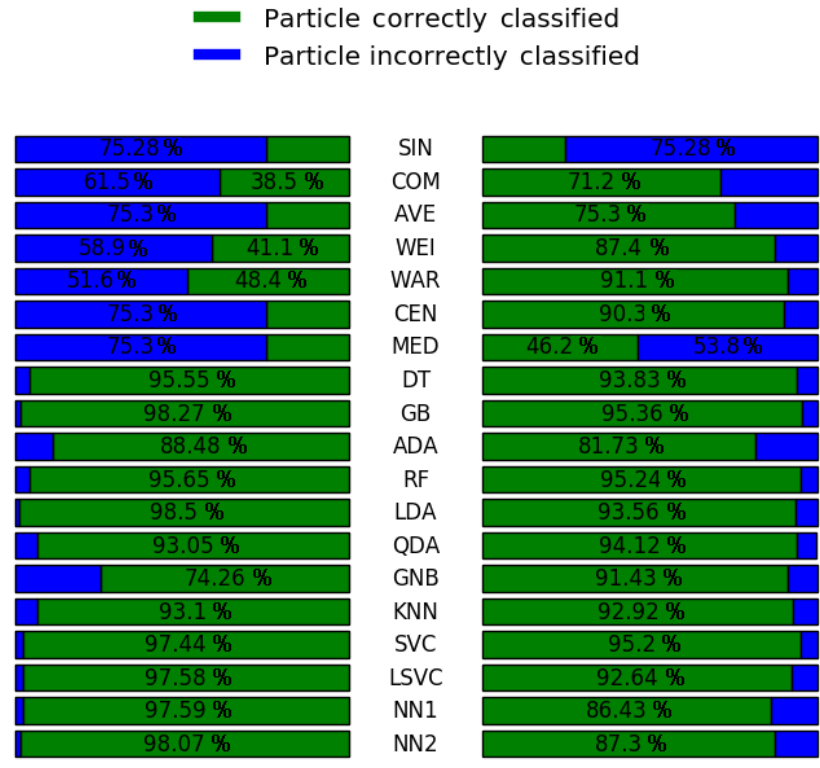

Figure 7. Performance of the different methods in terms of particles correctly classified for the mixed PSLs. The results on the left are for the full data set and on the right are the results for the reduced data set. Full names of each of the methods are given in Table 3.

achieved by using all the data in the HCA analysis (including both saturated and non-fluorescent material), for the laboratory data, it was beneficial to remove saturated and nonfluorescent material before conducting HCA analysis. Prefiltering was not applied to the supervised methods as explained in the Sect. 2. Only the best results are listed in Figs. 7 and 8; i.e. for the PSLs the results listed are from when all the particles were included and for the laboratory data the results obtained from the removal of non-fluorescent and fluorescent material are listed.

With the inclusion of the 1024 shape measurements we have a high-dimensional data set, without we have a relatively low-dimensional data set (nine dimensions). To give a good indication of the robustness of each algorithm to dimensionality as well as to ascertain whether the additional
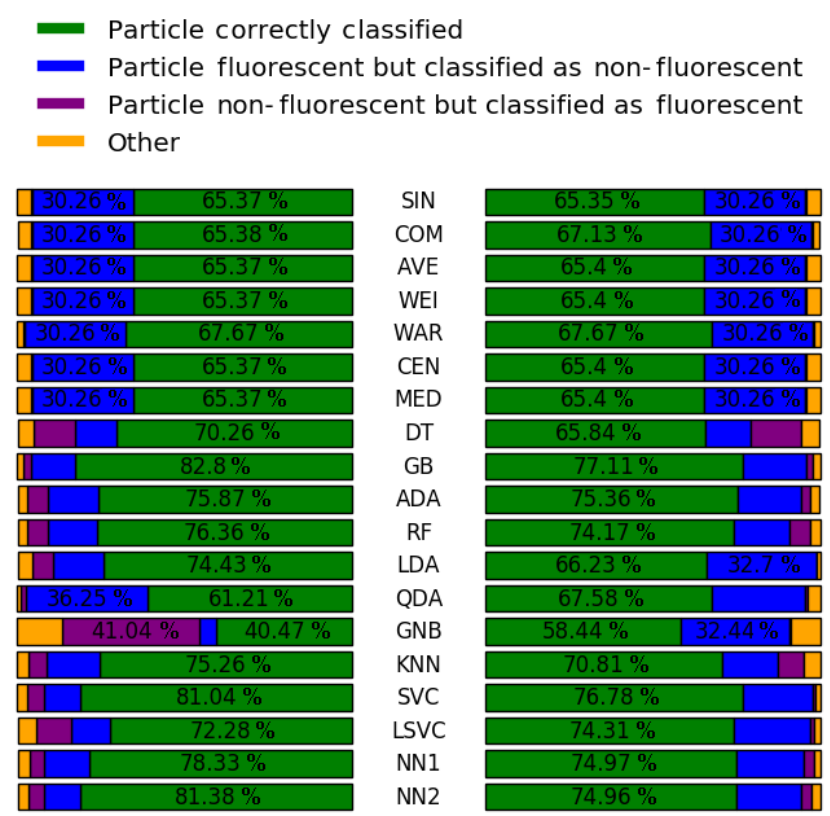

Figure 8. Performance of the different methods in terms of particles correctly classified and a breakdown of the errors for the laboratorygenerated aerosols. The results on the left are for the full data set and on the right are the results for the reduced data set. Full names of each of the methods are given in Table 3.

shape information yields any benefit, we provide results for both the full data set (1024 shape measurements, 8 fluorescent measurements and 1 size measurement) and the reduced data set (eight fluorescent measurements and one size measurement).

In Fig. 5 we see that the dye-doped PSLs should be highly separable by eye whereas in Fig. 6 it appears the laboratory data would present more of a challenge to the different algorithms. This is demonstrated also in our results where the percentage of data correctly classified for the laboratory data is in general much lower than that of the PSLs.

The exception to this is with HCA. For the full data the algorithm performs relatively poorly for both the PSLs and 
Table 3. Key for the shortened names for the different methods given in the figures.

\begin{tabular}{llr|llr}
\hline Key & Method & Subsection & Key & Method & Subsection \\
\hline SIN & HCA (single linkage) & 2.1 & RF & Random forest & 2.2 \\
COM & HCA (complete linkage) & 2.1 & LDA & Linear discriminant analysis & 2.3 \\
AVE & HCA (average linkage) & 2.1 & QDA & Quadratic discriminant analysis & 2.3 \\
WEI & HCA (weighted linkage) & 2.1 & GNB & Gaussian naïve Bayesian & 2.3 \\
WAR & HCA (ward linkage) & 2.1 & KNN & K Nearest-neighbour & 2.4 \\
CEN & HCA (centroid linkage) & 2.1 & SVC & Support vector classification & 2.5 \\
MED & HCA (median linkage) & 2.1 & LSVC & Linear support vector classification & 2.5 \\
DT & Decision tree & 2.2 & NN1 & Neural network - 1 Layer & 2.6 \\
GB & Gradient boosting & 2.2 & NN2 & Neural network - 2 Layers & 2.7 \\
ADA & AdaBoost & 2.2 & & & \\
\hline
\end{tabular}

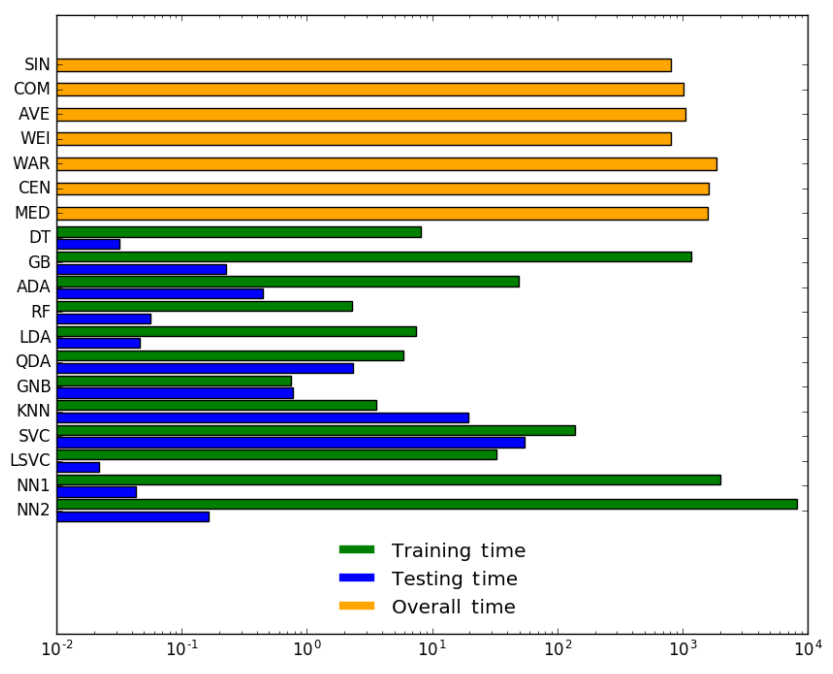

Figure 9. Performance of the different methods in terms of the time it takes for the method to train and test for the mixed PSLs.

the laboratory-generated aerosol. However, since we have already placed the non-fluorescent material and saturated material into groups on their own prior to analysis for the laboratory-generated aerosol, we see better performance for the laboratory data compared to the PSLs in this case. For the reduced data, however, we can yield generally good performance for the PSLs using the Ward linkage, but for the laboratory data the performance is generally poorer compared with the supervised methods regardless of whether the shape information is included.

Should these methods be applied to real-time applications, we would expect the testing data to contain much larger number of particles compared to the training data. For example, we could collect between $10^{4}$ and $10^{5}$ particles of laboratory data for training. However, over the space of several months in an ambient contain we might collect $10^{6}$ particles or more. It is for this reason we conclude that methods such as the ensemble methods and neural networks offer distinct advantages over HCA. For HCA the time requirements increase at a much faster rate than the number of particles (Müllner,
2011). In other words a doubling in the amount of data will result in more than a doubling in the amount of time required. A similar behaviour is true for the full support vector machines classifier.

The behaviour of the neural networks and the ensemble methods is much more desirable. While the training times are relatively large compared to other methods, once the model is fitted the testing time requirements are under a second for several thousand particles, which is much faster than the maximum count rate of the instrument.

Some of the methods, in particular the cluster analysis and the QDA perform poorer or equally as poor when the shape information is included. This is a reflection of the methods' ability to utilise high-dimensional data effectively rather on the instrument itself. In the case of HCA we would suggest that further research needs to be conducted in order to reduce the dimensionality of the data without losing information from the shape channels before using this method for the MBS. For QDA, the difficulty is in approximating the covariance matrix when the number of samples is less or similar to the number of dimensions. We would hence expect this method to perform better as larger samples are collected.

Decision trees and ensemble methods appear to be relatively robust to the introduction of the higher-dimensional data. This is to be expected since most of the methods undergo some kind of variable selection. Gradient boosting, however, does seem to offer improvements on the AdaBoost algorithm and random forests seem to improve on decision trees as is suggested in the literature.

Overall the best performing method was LDA for the PSLs and for laboratory-generated aerosols the gradient boosting algorithm performed better. Note, however, that gradient boosting only classified $0.23 \%$ less of the data correctly than LDA in the case of the PSLs data so overall our results indicate that gradient boosting is the best performing algorithm.

A possible alternative to gradient boosting is the twolayer neural network that performs nearly as well as gradient boosting for the full data set. It may be possible to extend the number of layers in order to yield further improvements but we would suggest, due to the increased time requirements 
with additional layers, that this should be done using a graphics processing unit (GPU) which could offer significant gains in the amount of time required to train the network. This is a benefit over the gradient boosting algorithm, where it is far less clear how the algorithm might be parallelised.

In contrast, neural networks are very difficult for the user to tailor to achieve good performance. The results presented are achieved after a lot of experimentation, especially in terms of the learning rate. A learning rate that is too high often will overshoot a minimum for the loss function and a learning rate that is too low will fail to reach a minimum at all. Overfitting, where the model fits very well for the training data but does not generalise well for the testing data, is also an issue. Overfitting seems not to be a problem for the gradient boosting algorithm, which also did not require any parameter selection by the user.

\subsection{Further analysis for the gradient boosting algorithm}

Due to the number of methods tested it is not practical to provide detailed information on all methods; instead we provide additional analysis for the gradient boosting algorithm which we found to offer, in general, better performance than the other methods. In particular we provide a further breakdown of the error term in Sect. 5.2.1. In Sect. 5.2.2 we split the laboratory data into individual samples and repeat the analysis. Finally in Sect. 5.2.3, we investigate the importance of the variables and the implication of removal of lesser important variables.

\subsubsection{Breakdown of error}

We can see in Figs. 7 and 8 that even for gradient boosting we still have a significant error in the classification rate. We therefore have elected to further break this error down into the different classes in Table 4. What we can see is that not only is a large proportion of the error due to fluorescent material being misclassified as non-fluorescent but the fungal spore sample is a particularly large source of error. However, amongst the material that was classified as fluorescent misclassification between the fluorescent classes is relatively small; e.g. only 67 fungal spores have been misclassified as bacteria.

As further samples are collected, especially in the case of fungal spores, we would hope these errors will start to decrease. However, an ongoing issue with the technique appears to be that a significant amount of particles within the fluorescent samples are weakly fluorescent and hence are difficult to classify correctly.

\subsubsection{Classification of individual species}

Our analysis in Sect. 5.1 only considers the broad biological classes: bacteria, fungal spores and pollen. In this subsection we enhance this analysis to split the bacteria into the three
Table 4. Breakdown of error for the gradient boosting algorithm on the full data set. For example, in the third row and first column we can see 31 particles of the bacteria sampled were classified as fungal spores.

\begin{tabular}{lrrrr}
\hline & Bacteria & $\begin{array}{r}\text { Fungal } \\
\text { spores }\end{array}$ & Pollen & $\begin{array}{r}\text { Non- } \\
\text { fluorescent }\end{array}$ \\
\hline Bacteria & 4637 & 67 & 66 & 174 \\
Fungal spores & 24 & 993 & 72 & 214 \\
Pollen & 31 & 58 & 1382 & 61 \\
Non-fluorescent & 547 & 1489 & 456 & 8679 \\
\hline
\end{tabular}

individual samples (i) washed BG spores, (ii) unwashed BG spores and (iii) unwashed E. coli. Similarly the pollen samples are also split up into (i) poplar pollen, (ii) aspen pollen and (iii) paper mulberry.

From Table 5 we can see that in the case of the bacterial samples we can effectively differentiate the washed sample from the unwashed samples. Distinguishing between the $E$. coli and the BG spores is also possible but with lesser success. It is entirely possible to distinguish between the paper mulberry and the other pollen samples but not between the aspen and poplar pollen sample. This is to be expected as the paper mulberry particles are significantly different from the aspen and poplar pollen samples, but the differences between the aspen and poplar pollen are very small.

Across all samples, excluding the particles which are incorrectly classified as non-fluorescent, when particles are misclassified they are most likely to be misclassified as a different sample from the same broad biological classes (bacteria, fungal spores and pollen). For example for the BG spores (w) sample, excluding the material that is misclassified as non-fluorescent, the largest misclassification is from particles being misclassified as E. coli (uw), which is still bacteria.

\subsubsection{Variable importance}

Since the instrument presented offers more information than the WIBS for example, it seems necessary to evaluate which of the variables presented offers the most information and how much of an impact removing lesser important variables has, on both time and the percentage of particles correctly classified.

It is possible to evaluate the performance of variables using the ensemble methods, e.g. gradient boosting. This is because each of the decision trees that come together to form the ensemble identifies which variables are best to split during the training stage. It is therefore possible to find out how often each of the variables are used to produce a split and this will give an indication of how important each of the variables are.

To determine which variables are most important for distinguishing between the different types of broad bio-type classes, we remove each of the fluorescent classes one by one 
Table 5. Further breakdown of error for the gradient boosting algorithm on the full data set. The abbreviations (w) and (uw) are used for washed and unwashed samples respectively.

\begin{tabular}{lrrrrrrrr}
\hline & $\begin{array}{r}\text { BG spores } \\
(\mathrm{w})\end{array}$ & $\begin{array}{r}\text { BG spores } \\
\text { (uw) }\end{array}$ & $\begin{array}{r}\text { E. coli } \\
\text { (uw) }\end{array}$ & Puffballs & $\begin{array}{r}\text { Paper } \\
\text { mulberry }\end{array}$ & $\begin{array}{r}\text { Aspen } \\
\text { pollen }\end{array}$ & $\begin{array}{r}\text { Poplar } \\
\text { pollen }\end{array}$ & $\begin{array}{r}\text { Non- } \\
\text { fluorescent }\end{array}$ \\
\hline BG spores (w) & 1127 & 12 & 71 & 5 & 0 & 4 & 2 & 39 \\
BG spores (uw) & 4 & 1373 & 197 & 19 & 6 & 9 & 16 & 27 \\
E. coli (uw) & 79 & 227 & 1385 & 20 & 6 & 8 & 15 & 41 \\
Puffballs & 3 & 16 & 13 & 1082 & 10 & 35 & 62 & 254 \\
Paper mulberry & 0 & 5 & 3 & 15 & 998 & 2 & 2 & 11 \\
Aspen pollen & 2 & 7 & 3 & 13 & 2 & 109 & 65 & 22 \\
Poplar pollen & 1 & 8 & 2 & 23 & 1 & 50 & 68 & 20 \\
Non-fluorescent & 201 & 183 & 317 & 1430 & 18 & 249 & 239 & 8714 \\
\hline
\end{tabular}

and train the algorithm on the subset of the data and record the importance of each of the variables. In Fig. 10 we show the importance of the variables for the full data, bacteria vs. fungal spores (pollen removed), bacteria vs. pollen (fungal spores removed) and finally fungal spores vs. pollen (bacteria removed). The top three most important variables always contained a selection of four different variables, so we provide the importance for these four variables in Fig. 10.

Finally, we remove all but the top 512, 256, 128, .., 2 variables and repeat the analysis. The total time required and the percentage of particles classified correctly are given in Fig. 11.

What we can see from our analysis is that for the data set with none of the broad biological classes (bacteria, pollen and fungal spores) removed the most important variable is the Size followed by the third fluorescent channel. For the remaining subsets of the data tested the most important variables are a fluorescent variable followed by the size. From Fig. 11, however, we can see that four variables alone, while the most important, are not sufficient to maintain the results possible with the full data set. Instead, reduction in the number of variables does necessarily lead to a reduction in performance in someway. Nonetheless the decrease from 1024 variables to 128 produces a very small decrease in performance and hence a smaller shape detector may be sufficient.

\section{Conclusions}

Ultraviolet light-induced fluorescence (UV-LIF) is becoming a widely used and accepted method for collecting fluorescent signatures for bioaerosols. However, the applicability of the method has yet to be demonstrated for routine real-time monitoring and reporting applications for airborne biological particles. In this paper we have combined the well-developed and researched field of machine learning with the application of identifying atmospheric aerosol. We have demonstrated that previously used unsupervised methods may not be best at discriminating between aerosol using single particle broadband UV-LIF spectrometers and using the MBS we
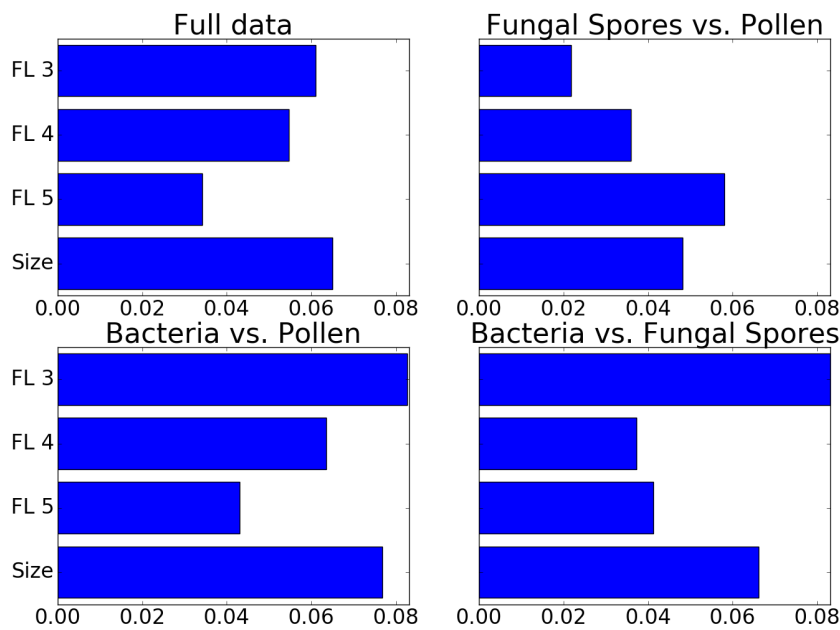

Figure 10. Variable importance for the different subsets of the data.

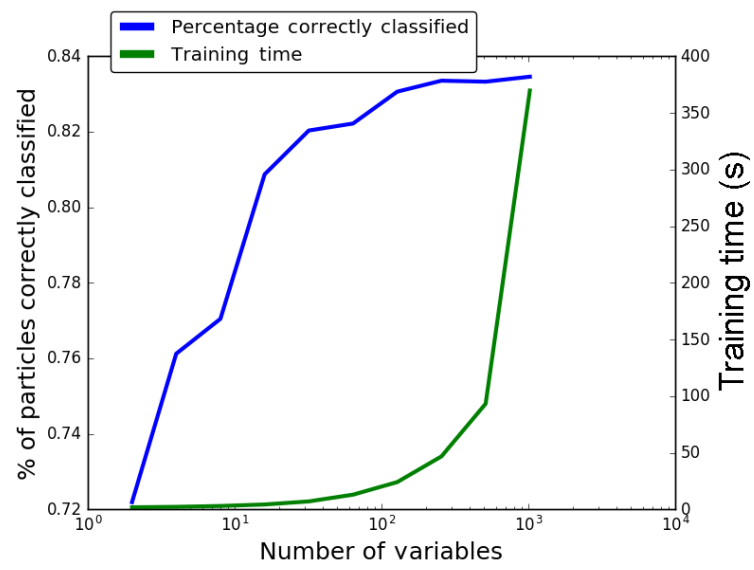

Figure 11. Variable importance for the different subsets of the data.

have identified the gradient boosting classifier as a possible supervised alternative.

We have tested a variety of different methods that could be used for discriminating between different types 
of bioaerosol. Cluster analysis, while working well for the reduced data set for PSLs, seems to struggle for the laboratory-generated aerosol and when applied to the higherdimensional data set, so we suggest that more research for this method is required before it could be reasonably used on ambient data collected using the MBS. For the Gaussian methods it seems that the methods work reasonably well for the PSLs, but we believe there are better alternatives when discriminating between atmospheric aerosol.

For the $k$-nearest neighbours method we believe that a limiting factor is in the time it takes for the method to classify the testing data. Similarly, while the full support vector machine performs very well, the time requirements would be inappropriate when larger samples are collected. Conversely, while the linear version of the support vector machine performs much faster, it is at the cost of performance, so we suggest that support vector machines not be used for this task.

Overall, the method we suggest for classification of atmospheric aerosol is the gradient boosting algorithm which produces the best results with limited user input but has the drawback that it cannot easily be parallelised. Another possible alternative in the future, once more research is conduced, is the neural network, which can be easily ported to a GPU for substantial speedup in training but requires a much larger input for the user and produces slightly worse results compared to the gradient boosting algorithm.

From our further analysis of the gradient boosting algorithm we also see that a disadvantage for the data we have collected is in the sample of fungal spores, which is often misclassified as non-fluorescent since a good proportion of the particles is weakly fluorescent. We believe this issue can be circumvented with collection of a wider range of fungal spore samples in the future. Also, we see that for the MBS we have reasonable success in discriminating between single bacterial samples.

Finally we realise that performance can be maintained while removing a reasonable number of the lesser important variables, leading us to conclude that a smaller shape detector may be sufficient.

Since these supervised learning algorithms have yet to be applied to the data produced using the WIBS it is not currently possible to draw any clear conclusions as to the performance of the MBS versus the WIBS. Instead the authors suggest that to provide direct comparison, further research needs to be undertaken whereby both instruments are used for identical samples.

\section{Data availability}

The data used to formulate the results in the paper can be provided upon request by contacting the first author, using the correspondence e-mail address (simon.ruske@ postgrad.manchester.ac.uk).
Acknowledgements. Simon Ruske is funded by a NERC grant (NE/L002469/1) as part of the Manchester-Liverpool Doctoral Training Partnership. The MBS instrument was funded by the NERC research grant, NE/K006002/1 "Ice Nucleation Process Investigation and Quantification".

Edited by: F. Pope

Reviewed by: D. Baumgardner and one anonymous referee

\section{References}

Breiman, L.: Bagging predictors, Mach. Learn., 24, 123-140, 1996. Breiman, L.: Random forests, Mach. Learn., 45, 5-32, 2001.

Cortes, C. and Vapnik, V.: Support-vector networks, Mach. Learn., 20, 273-297, 1995.

Crawford, I., Bower, K. N., Choularton, T. W., Dearden, C., Crosier, J., Westbrook, C., Capes, G., Coe, H., Connolly, P. J., Dorsey, J. R., Gallagher, M. W., Williams, P., Trembath, J., Cui, Z., and Blyth, A.: Ice formation and development in aged, wintertime cumulus over the UK: observations and modelling, Atmos. Chem. Phys., 12, 4963-4985, doi:10.5194/acp-12-4963-2012, 2012.

Crawford, I., Robinson, N. H., Flynn, M. J., Foot, V. E., Gallagher, M. W., Huffman, J. A., Stanley, W. R., and Kaye, P. H.: Characterisation of bioaerosol emissions from a Colorado pine forest: results from the BEACHON-RoMBAS experiment, Atmos. Chem. Phys., 14, 8559-8578, doi:10.5194/acp-14-8559-2014, 2014.

Crawford, I., Ruske, S., Topping, D. O., and Gallagher, M. W.: Evaluation of hierarchical agglomerative cluster analysis methods for discrimination of primary biological aerosol, Atmos. Meas. Tech., 8, 4979-4991, doi:10.5194/amt-8-4979-2015, 2015.

Cziczo, D. J., Froyd, K. D., Hoose, C., Jensen, E. J., Diao, M., Zondlo, M. A., Smith, J. B., Twohy, C. H., and Murphy, D. M.: Clarifying the dominant sources and mechanisms of cirrus cloud formation, Science, 340, 1320-1324, 2013.

Freund, Y. and Schapire, R. E.: A desicion-theoretic generalization of on-line learning and an application to boosting, in: Computational learning theory, Springer, 23-37, 1995.

Gurian-Sherman, D. and Lindow, S. E.: Bacterial ice nucleation: significance and molecular basis, FASEB J., 7, 1338-1343, 1993.

Hader, J. D., Wright, T. P., and Petters, M. D.: Contribution of pollen to atmospheric ice nuclei concentrations, Atmos. Chem. Phys., 14, 5433-5449, doi:10.5194/acp-14-5433-2014, 2014.

Healy, D. A., Huffman, J. A., O'Connor, D. J., Pöhlker, C., Pöschl, U., and Sodeau, J. R.: Ambient measurements of biological aerosol particles near Killarney, Ireland: a comparison between real-time fluorescence and microscopy techniques, Atmos. Chem. Phys., 14, 8055-8069, doi:10.5194/acp-14-80552014, 2014.

Herich, H., Gianini, M., Piot, C., Močnik, G., Jaffrezo, J.-L., Besombes, J.-L., Prévôt, A., and Hueglin, C.: Overview of the impact of wood burning emissions on carbonaceous aerosols and PM in large parts of the Alpine region, Atmos. Environ., 89, 6475,2014

Hernandez, M., Perring, A. E., McCabe, K., Kok, G., Granger, G., and Baumgardner, D.: Chamber catalogues of optical and fluorescent signatures distinguish bioaerosol classes, Atmos. Meas. Tech., 9, 3283-3292, doi:10.5194/amt-9-3283-2016, 2016. 
Hoose, C. and Möhler, O.: Heterogeneous ice nucleation on atmospheric aerosols: a review of results from laboratory experiments, Atmos. Chem. Phys., 12, 9817-9854, doi:10.5194/acp-12-98172012, 2012.

Hsu, C., Chang, C., and Lin, C.: A practical guide to support vector classification, Department of Computer Science and Information Engineering, National Taiwan University, Taipei, Taiwan, 2003.

Huffman, J. A., Prenni, A. J., DeMott, P. J., Pöhlker, C., Mason, R. H., Robinson, N. H., Fröhlich-Nowoisky, J., Tobo, Y., Després, V. R., Garcia, E., Gochis, D. J., Harris, E., Müller-Germann, I., Ruzene, C., Schmer, B., Sinha, B., Day, D. A., Andreae, M. O., Jimenez, J. L., Gallagher, M., Kreidenweis, S. M., Bertram, A. K., and Pöschl, U.: High concentrations of biological aerosol particles and ice nuclei during and after rain, Atmos. Chem. Phys., 13, 6151-6164, doi:10.5194/acp-13-6151-2013, 2013.

Hummel, M., Hoose, C., Gallagher, M., Healy, D. A., Huffman, J. A., O'Connor, D., Pöschl, U., Pöhlker, C., Robinson, N. H., Schnaiter, M., Sodeau, J. R., Stengel, M., Toprak, E., and Vogel, H.: Regional-scale simulations of fungal spore aerosols using an emission parameterization adapted to local measurements of fluorescent biological aerosol particles, Atmos. Chem. Phys., 15, 6127-6146, doi:10.5194/acp-15-6127-2015, 2015.

Jacobson, M. Z. and Streets, D. G.: Influence of future anthropogenic emissions on climate, natural emissions, and air quality, J. Geophys. Res.-Atmos., 114, D08118, doi:10.1029/2008JD011476, 2009.

Jia, Y., Shelhamer, E., Donahue, J., Karayev, S., Long, J., Girshick, R., Guadarrama, S., and Darrell, T.: Caffe: Convolutional Architecture for Fast Feature Embedding, Proceedings of the 22nd ACM international conference on Multimedia, Orlando, Florida, USA, 3-7 November 2014, 675-678, 2014.

Joly, M., Attard, E., Sancelme, M., Deguillaume, L., Guilbaud, C., Morris, C. E., Amato, P., and Delort, A.-M.: Ice nucleation activity of bacteria isolated from cloud water, Atmos. Environ., 70, 392-400, 2013.

Kaye, P., Stanley, W., Hirst, E., Foot, E., Baxter, K., and Barrington, S.: Single particle multichannel bio-aerosol fluorescence sensor, Opt. Express, 13, 3583-3593, 2005.

Kaye, P. H.: Spatial light-scattering analysis as a means of characterizing and classifying non-spherical particles, Meas. Sci. Technol., 9, 141-149, 1998.

Kaye, P. H., Alexander-Buckley, K., Hirst, E., Saunders, S., and Clark, J.: A real-time monitoring system for airborne particle shape and size analysis, J. Geophys. Res.-Atmos., 101, 1921519221, 1996.

Kennedy, R. and Smith, M.: Effects of aeroallergens on human health under climate change, in: Health Effects of Climate Change in the UK 2012, edited by: Vardoulakis, S. and Heaviside, C., 83-96, 2012.
LeCun, Y., Bottou, L., Bengio, Y., and Haffner, P.: Gradient-based learning applied to document recognition, Proceedings of the IEEE, 86, 2278-2324, 1998.

Möhler, O., DeMott, P. J., Vali, G., and Levin, Z.: Microbiology and atmospheric processes: the role of biological particles in cloud physics, Biogeosciences, 4, 1059-1071, doi:10.5194/bg-4-10592007, 2007.

Morris, C. E., Conen, F., Alex Huffman, J., Phillips, V., Pöschl, U., and Sands, D. C.: Bioprecipitation: a feedback cycle linking Earth history, ecosystem dynamics and land use through biological ice nucleators in the atmosphere, Glob. Change Biol., 20, 341-351, 2014.

Müllner, D.: Modern hierarchical, agglomerative clustering algorithms, available at: https://arxiv.org/abs/1109.2378, 2011.

Müllner, D.: fastcluster: Fast hierarchical, agglomerative clustering routines for R and Python, J. Stat. Softw., 53, 1-18, 2013.

Pedregosa, F., Varoquaux, G., Gramfort, A., Michel, V., Thirion, B., Grisel, O., Blondel, M., Prettenhofer, P., Weiss, R., Dubourg, V., Vanderplas, J., Passos, A., Cournapeau, D., Brucher, M., Perrot, M., and Duchesnay, E.: Scikit-learn: Machine Learning in Python, J. Mach. Learn. Res., 12, 2825-2830, 2011.

Prenni, A., Tobo, Y., Garcia, E., DeMott, P., Huffman, J., McCluskey, C., Kreidenweis, S., Prenni, J., Pöhlker, C., and Pöschl, U.: The impact of rain on ice nuclei populations at a forested site in Colorado, Geophys. Res. Lett., 40, 227-231, 2013.

Robinson, N. H., Allan, J. D., Huffman, J. A., Kaye, P. H., Foot, V. E., and Gallagher, M.: Cluster analysis of WIBS single-particle bioaerosol data, Atmos. Meas. Tech., 6, 337-347, doi:10.5194/amt-6-337-2013, 2013.

Schumacher, C. J., Pöhlker, C., Aalto, P., Hiltunen, V., Petäjä, T., Kulmala, M., Pöschl, U., and Huffman, J. A.: Seasonal cycles of fluorescent biological aerosol particles in boreal and semiarid forests of Finland and Colorado, Atmos. Chem. Phys., 13, 11987-12001, doi:10.5194/acp-13-11987-2013, 2013.

Spracklen, D. V. and Heald, C. L.: The contribution of fungal spores and bacteria to regional and global aerosol number and ice nucleation immersion freezing rates, Atmos. Chem. Phys., 14, 90519059, doi:10.5194/acp-14-9051-2014, 2014.

Tobo, Y., Prenni, A. J., DeMott, P. J., Huffman, J. A., McCluskey, C. S., Tian, G., Pöhlker, C., Pöschl, U., and Kreidenweis, S. M.: Biological aerosol particles as a key determinant of ice nuclei populations in a forest ecosystem, J. Geophys. Res.-Atmos., 118, 10100-10110, doi:10.1002/jgrd.50801, 2013.

Toprak, E. and Schnaiter, M.: Fluorescent biological aerosol particles measured with the Waveband Integrated Bioaerosol Sensor WIBS-4: laboratory tests combined with a one year field study, Atmos. Chem. Phys., 13, 225-243, doi:10.5194/acp-13225-2013, 2013. 